\title{
Éditorial: Problèmes de santé des femmes âgées*
}

En 1995, le Rapport mondial sur le développement humain des Nations Unies classait le Canada comme le meilleur endroit pour vivre pour un homme. Pour les femmes, cependant, le Canada ne vient qu'en neuvième place (Nations Unies, 1995). Selon ce rapport, «les femmes n'ont les mêmes possibilités que les hommes dans aucune sociétés. Bien que l'attention portée aux questions féminines, et en particulier à leur santé, ait augmenté au cours des dernières années, l'information reste quand même relativement peu abondante en ce qui concerne la distribution, les causes et les conséquences des pratiques et des problèmes de santé des femmes âgées au Canada, qu'elles vivent dans leur milieu ou dans un établissement. Le point est particulièrement intéressant lorsqu'on considère l'augmentation rapide de la proportion de femmes âgées au Canada. Par exemple, en 1991, 13 pour cent des femmes canadiennes (1,9 million) étaient âgées de plus de 65 ans, et on estime que cette proportion passera à 18 pour cent d'ici 2016 , et à 25 pour cent d'ici 2041. Encore plus important, les chiffres montrent que l'augmentation la plus rapide se fera chez les femmes les plus âgées, soit celles qui ont 85 ans ou plus (Statistiques Canada, 1995).

Compte tenu de l'espérance de vie plus grande des femmes, il n'est pas surprenant de constater que la plupart des problèmes liés au vieillissement, comme l'augmentation du risque d'usage de médicaments multiples (polypharmacie), de maladies chroniques concurrentes (c.-à-d. maladies cardiaques, arthrite, rhumatismes, ostéoporose, cancer), d'invalidité et de chutes aient été présentés comme des problèmes féminins (Gee \& Kimball, 1987). Les femmes âgées sont également à plus grand risque d'institutionnalisation, et semblent présenter une fréquence plus élevée de dépression et d'autres problèmes de santé mentale associés à l'avancement en âge et à l'évolution de la santé physique (c.-à-d. la solitude liée à la perte du conjoint, les problèmes de mémoire associés à l'usage de médicaments) que les hommes du même âge (Santé Canada, 1993). Malheureusement, notre compréhension des risques de santé des femmes âgées et les domaines d'intervention sont limités par le manque de recherche dans le domaine et l'insuffisance de connaissances sur le rôle des soins de santé et des facteurs cognitifs, psychologiques et socioculturels dans la facilitation de comportements de santé positifs chez les femmes âgées. Chacune de ces questions est étudiée tour à tour. 


\section{Usage de médicaments multiples (polypharmacie)}

Plusieurs études épidémiologiques réalisées au Canada et dans d'autres pays (Campbell, Spears, \& Borrie, 1990; Chrischilles et al., 1992; Graham, Carver, \& Brett, 1995) ont démontré l'une après l'autre un taux élevé de consommation de médicaments et de polypharmacie parmi les personnes âgées, les femmes en particulier. Les estimations de fréquence pour les médicaments sur ordonnance et en vente libre liés à la gestion de la douleur et aux problèmes de sommeil ou d'anxiété sont considérablement plus élevées que celles qu'on retrouve chez les hommes, dans tous les groupes d'âge. Le taux plus élevé de prise de médicaments psychotropes chez les femmes signifie soit un plus grand empressement des femmes à prendre ces médicaments ou un plus grand empressement des médecins à les prescrire aux femmes. Dans l'étude menée par Campbell et al. (1990), 32 pour cent des femmes âgées recevaient des médicaments psychotropes, comparativement à 16,2 pour cent des hommes. Les auteurs notent que les femmes commençaient habituellement à recevoir des psychotropes dans une période de deuil et qu'elles sont plus susceptibles d'avoir perdu un conjoint.

Une préoccupation importante concernant l'utilisation relativement plus élevée de certaines classes de médicaments chez les femmes âgées est leur plus grande propension aux réactions adverses aux médicaments. Les femmes âgées pourraient être plus susceptibles que les hommes de faire une réaction aux médicaments (sédation, désorientation, chutes, ulcères peptiques et saignements gastro-intestinaux) pour plusieurs raisons, qui n'ont pas fait l'objet d'études poussées:

- un risque accru d'utilisation de médicaments multiples et de co-morbidité;

- un risque accru d'altération de la pharmacocinétique (absorption, distribution et élimination) et de la pharmacodynamique (réaction) des médicaments en raison d'un poids corporel moindre, de l'exposition à l'hormonothérapie, des modifications de la composition corporelle (plus grand pourcentage de gras corporel, plus faible volume d'eau), des changements métaboliques, de la polypharmacie et de la co-morbidité,

- une plus grande possibilité d'amoindrissement des réserves physiologiques requises pour compenser les perturbations amenées par les médicaments dans l'homéostasie normale.

Il y a aussi de plus en plus de preuves que la réaction à certains médicaments (bétabloquants, psychotropes et neuroleptiques) peut différer selon l'origine ethnique (Merkatz et al., 1993), ce qui illustre l'importance qu'il y a à considérer l'origine ethnique des femmes âgées dans la pratique de prescription. 


\section{Maladies chroniques et infirmités}

Bien que le cancer, et en particulier le cancer du sein, ait pris le premier plan dans les questions de santé féminine, ce sont les maladies cardio-vasculaires et les désordres des vaisseaux sanguins qui constituent le principal problème de santé des femmes âgées. L'importance des maladies cardiaques chez les femmes âgées devient évident lorsqu'on considère le nombre total de personnes atteintes et les taux de mortalité et de morbidité qui y sont associés. Aux États-Unis, on estime qu'une femme sur deux peut s'attendre à souffrir de problèmes cardiaques graves comparativement à une sur neuf pour le cancer du sein (Healy, 1995). De plus, on compte annuellement 245,000 décès dûs aux maladies cardiaques, comparativement à 46,000 pour le cancer du sein. Les statistiques canadiennes démontrent de même que dans l'ensemble, un nombre beaucoup plus grand de femmes âgées meurent de maladies cardiaques que du cancer du sein. Malgré cela, aucune étude à grande échelle n'a été mise en branle pour étudier les différences liées au sexe dans le risque et la prévention des maladies cardiaques chez les personnes âgées, malgré que l'on reconnaisse que les maladies cardiaques peuvent être prévenues.

Bien que l'étude de Framingham ait fourni des données importantes sur les principaux facteurs de risque de maladies cardio-vasculaires (tabagisme, hypertension, obésité, diabète) qui peuvent être appliqués aux deux sexes, l'étude perpétue malheureusement la croyance selon laquelle les femmes ne souffrent pas de maladies coronaires et que leurs plaintes de douleurs à la poitrine sont sans fondement (Healy, 1995). De plus, les conclusions fondées sur l'âge dans les essais cliniques de médicaments et d'autre thérapies pour les maladies cardiaques (Gurwitz, Col, \& Avorn, 1992) ont entraîné une sous-représentation des femmes âgées dans l'étude, et donc un manque de données sur les avantages et les risques de telles thérapies chez les femmes âgées. Ces croyances erronées et ces limites des études ont directement affecté la façon dont les femmes âgées avec des problèmes cardio-vasculaires sont traitées dans le système de santé (Beery, 1995). Par exemple,

- les femmes souffrant de douleurs à la poitrine et présentant des résultats de tests anormaux sont moins susceptibles d'être soumises à des tests approfondis que les hommes;

- les femmes présentant des douleurs à la poitrine sont moins susceptibles de recevoir des traitements variés (par. ex., bétabloquants, anticoagulants) et des interventions effractives que les hommes;

- les femmes ont typiquement été exclues des recherches sur le rôle des facteurs de risque dans le développement et la prévention des maladies cardiaques (c.-à-d. cholestérol LDL et HDL);

- le rationnement fondé sur l'âge des soins cardio-vasculaires que l'on remarque dans certains pays s'est traduit par un rationnement 
fondé sur le sexe, puisque les maladies cardiaques chez la femme apparaissent entre 10 et 20 ans plus tard que chez les hommes.

En ce qui concerne le statut fonctionnel et les limites à l'activité, les données tant canadiennes qu'américaines montrent une progression exponentielle avec l'âge dans la proportion de la population présentant des incapacités. Chose plus importante, ces données indiquent que le taux de fréquence de différentes incapacités est, de façon constante, plus élevé chez les femmes que chez les hommes, dans tous les groupes d'âge (Statistiques Canada, 1995). Comme les femmes vivent en moyenne plus longtemps que les hommes, elles sont plus susceptibles que ceux-ci de devoir vivre avec une incapacité durant une période plus longue, sans conjoint (et vraisemblablement sans accès à d'autres ressources sociales) pour les aider à vivre de façon indépendante dans la communauté. Bien que cela fasse encore l'objet d'un débat, certains chercheurs suggèrent que les différences sexuelles dans les incapacités sont le résultat d'artifices méthodologiques plutôt que de différences réelles dans la santé des hommes et des femmes (Gee \& Kimball, 1987). La question devra donc être clarifiée.

\section{Chutes}

Les chutes comptent pour une grande proportion de la morbidité et de la mortalité liées aux blessures dans tous les groupes d'âge, et plus particulièrement chez les personnes âgées. Des statistiques récentes recueillies au Canada et aux Etats-Unis montrent que les chutes sont la principale cause d'hospitalisation pour blessures et de décès accidentel chez les personnes de 65 ans ou plus (Riley, 1992). Plusieurs études ont démontré un risque plus élevé de chutes chez les femmes âgées que chez les hommes du même âge (Campbell et al., 1990). Les raisons possibles comprennent une plus grande répugnance des hommes à signaler une chute et une plus grande possibilité que les femmes âgées deviennent isolées, perdent de la force musculaire et utilisent plusieurs médicaments, en particulier des psychotropes. Les femmes âgées, outre qu'elles semblent présenter une fréquence de chutes plus élevée que les hommes, sont également plus susceptibles de subir des dommages sérieux aux tissus mous et des fractures (Sattin et al., 1990), ce dernier élément reflétant leur risque plus élevé d'ostéoporose.

Parmi les blessures associées aux chutes, les fractures de la hanche sont les plus fréquentes et celles qui sont associées aux risques d'hospitalisation et de décès les plus élevés (Kiel, 1993). En plus des blessures et de l'hospitalisation, les chutes et les fractures qui y sont associées peuvent également avoir des effets physiques et psychologiques à court et à long terme (Nevitt, Cummings, \& Hudes, 1991), qui peuvent être aggravés chez les femmes âgées lorsqu'on les associe à une co-morbidité plus élevée et au traitement différent accordé par le système de santé. Certains de ces effets peuvent comprendre: 
- la peur, l'anxiété et la dépression,

- une plus grande susceptibilité à des chutes ultérieures,

- l'isolation sociale,

- un affaiblissement de la santé et du statut fonctionnel,

- une perte d'indépendance et de confiance,

- une qualité de vie réduite, et

- la mort.

\section{La vie en établissement chez la femme}

La probabilité plus élevée pour les femmes de devoir être placée en établissement a été démontrée par plusieurs études au Canada et ailleurs (Young, Forbes, \& Hirdes, 1994). Il existe plusieurs facteurs qui contribuent à l'accroissement du risque pour les femmes, mais l'explication principale touche les interrelations entre les changements dans la santé et les changements dans les relations sociales au cours de la vie. Comme nous l'avons noté plus haut, l'espérance de vie plus grande chez les femmes de différents âges s'accompagne d'une plus grande probabilité de périodes d'incapacité, qui elles-mêmes durent plus longtemps.

Le taux de mortalité plus élevé des hommes d'âge comparable représente également un facteur de placement en établissement important chez les femmes âgées. Les hommes âgés dont les fonctions sont amoindries peuvent habituellement compter sur la présence d'un conjoint pour leur assurer un soutien dans les activités de la vie de tous les jours et les activités instrumentales de la vie de tous les jours. Cependant, la tendance des femmes à épouser des hommes plus âgés et les taux de mortalité plus élevés chez les hommes font que les femmes sont beaucoup plus susceptibles de connaître le veuvage (Martin Matthews, 1991). En conséquence, les femmes âgées sont plus susceptibles de vivre dans un état d'infirmité sans l'aide d'un conjoint pour les aider à vivre de façon autonome dans leur milieu. Bien que les enfants soient une source importante de soutien social aux veuves âgées, ils peuvent ne pas disposer des ressources suffisantes pour composer avec l'apparition d'incapacités importantes. De la même façon, l'accès à des services communautaires structurés qui peuvent réduire la fréquence de placement en établissement n'est pas disponible en quantité suffisante pour avoir un impact à grande échelle sur ces taux de placement.

Il est surprenant de constater que très peu d'études contemporaines ont été effectuées sur les aspects sociaux et psychologiques de la vie en établissement et qu'il existe encore moins de données sur la relation entre ces éléments et le sexe. Par exemple, il est possible que la probabilité plus élevée que les femmes âgées vivant en établissement n'aient pas de conjoint puisse avoir un effet délétère sur leur qualité de vie, l'accès aux soins de santé et les résultats sur leur santé. De façon plus particulière, comparativement aux personnes en établissement disposant d'un réseau social fort, 
celles qui n'en ont pas ou dont le réseau est peu étendu semblent présenter un risque plus élevé:

- d'affaiblissement du niveau d'autonomie,

- de bien-être psychologique moindre,

- de résultats de réhabilitation plus faibles,

- d'avoir un accès réduit à des bénévoles,

- de recevoir moins de services, et

- de mortalité

Comme les femmes sont plus nombreuses que les hommes dans les établissements pour personnes âgées (généralement dans une proportion de 3 à 1), les femmes âgées peuvent être à plus haut risque de résultats moindres que les hommes dans le même environnement, parce qu'elles sont plus susceptibles de vivre suffisamment longtemps pour connaître une réduction marquée de leur réseau social et subir la détérioration de la santé associée à l'avancement en âge. En même temps, on en sait très peu sur les effets positifs du placement en établissement, en particulier pour les femmes âgées qui vivent seules, sans soutien dans leur milieu.

\section{Santé mentale}

La tendance qu'ont les professionnels de la santé et le public en général à minimiser l'importance des problèmes de santé mentale (comme la dépression et l'anxiété) comme déterminants de la santé et de la qualité de vie chez les personnes âgées a été particulièrement préjudiciable aux femmes âgées. Ceci provient du fait que ces problèmes sont plus fréquents chez les femmes et augmentent avec plusieurs facteurs liés à l'âge, notamment la diminution du revenu, un soutien social insuffisant, les deuils et autres événements générateurs de stress, l'incapacité physique et l'utilisation des médicaments (Koenig \& Blazer, 1992). La maladie dépressive peut non seulement entraîner des effets négatifs aux plans émotif, social et économique, mais elle peut aussi aggraver, prolonger et précipiter des maladies physiques chez les femmes âgées en affectant différents systèmes physiologiques, les soins personnels et le suivi des traitements (Lyness et al., 1996).

Bien que la dépression soit généralement considérée comme pouvant être traitée chez les personnes âgées, la reconnaissance et le traitement des désordres dépressifs par les professionnels de la santé apparaît constamment comme relativement faible, en particulier chez les patients âgés et atteints d'incapacités plus profondes (Lyness et al., 1996). De plus, les données sur les personnes âgées vivant dans leur milieu indiquent que très peu de personnes âgées sollicitent une aide professionnelle en santé mentale (Koenig \& Blazer, 1992). Bien que ce sujet soit relativement inexploré chez les personnes âgées, il apparaît aussi que le stigmate social associé à la maladie mentale puisse se traduite par une plus grande répugnance dans certains sous-groupes culturels de femmes âgées à reconnaître ou à 
signaler les symptômes. Des études additionnelles seront nécessaires pour analyser le risque et les effets des problèmes mentaux et leur sous-traitement chez les femmes âgées.

Bien que les constats actuels sur les différences reliées au sexe dans la fréquence et l'incidence de la maladie d'Alzheimer et les désordres qui y sont reliés ne soient pas constants, l'augmentation exponentielle de la démence avec l'âge et l'espérance de vie plus grande chez les femmes laissent supposer une plus grande probabilité pour les femmes âgées d'être touchées par des problèmes de démence (Santé Canada, 1993). De plus, compte tenu de leur prédominance parmi les soignants, ce sont les femmes, incluant les femmes âgées, plutôt que les hommes qui ont tendance à devoir assumer la fardeau des soins et les conséquences sur la santé physique et émotionnelle qui sont associées au fait de devoir prendre soin de personnes atteintes de désordres démentiels.

\section{Comportements préventifs en matière de santé}

Malgré les efforts considérables consentis pour examiner la contribution des comportements de prévention en matière de santé chez les femmes jeunes et d'âge moyens, (c.-à-d. l'exercice physique, la nutrition, le tabagisme, le dépistage du cancer), la pertinence de ces comportements pour la santé et le bien-être des femmes âgées est moins claire et fait l'objet d'un débat animé. Les grands travaux de recherche sur les facteurs de risque et l'efficacité des activités de promotion de la santé et de prévention des maladies ont généralement exclu les personnes âgées, et les femmes âgées en particulier. En conséquence, une proportion substantielle des femmes à risque plus élevé d'impacts négatifs sur la santé, soit celles qui appartiennent aux groupes d'âges les plus élevés, ne sont pas touchées par les lignes directrices actuelles en matière d'interventions appropriées de prévention. L'absence relative de données sur les risques associés aux pratiques de santé déficientes chez les personnes âgées et les bénéfices potentiels des initiatives de prévention chez les personnes de plus de 65 ans dépend largement de la tendance générale à mettre l'accent sur les populations plus jeunes pour tout ce qui concerne les mesures primaires de prévention. On peut illustrer ce point par la controverse actuelle sur l'à-propos du dépistage du cancer du sein chez les femmes âgées. On a remarqué que la plupart des études marquantes sur les avantages du dépistage par mammographie ne comprenaient pas les femmes âgées (Costanza, 1994), malgré qu'il soit évident que la maladie frappe d'une façon disproportionnée les femmes des groupes d'âges plus avancés.

Le défaut de reconnaître les avantages potentiels des différentes stratégies de prévention pour les femmes âgées est malheureux à la lumière de l'augmentation des faits tendant à démontrer que les femmes âgées peuvent bénéficier d'une amélioration importante de leur santé et de leur qualité de vie en modifiant leurs habitudes de vie et en adoptant 
des pratiques de prévention de la maladie, entre autres en augmentant leur niveau d'exercice physique, en améliorant leur alimentation et en cessant de fumer (Bravo et al., 1996; Maxwell \& Hirdes, 1993; Rosenberg, Palmer, \& Shapiro, 1990).

Outre le manque de recherches, d'autres barrières ont contribué à l'insuffisance ou à l'impropriété des activités de recherche et des activités cliniques sur les pratiques de prévention de la maladie chez les femmes âgées:

- le défaut de reconnaître que les femmes âgées constituent un groupe extrêmement hétérogène sur le plan des caractéristiques psychosociales, culturelles et physiques;

- la tendance, chez les professionnels de la santé et le public en général, à catégoriser les questions de santé des personnes âgées, et plus particulièrement des femmes âgées, comme des "problèmes de santé qui ne sont pas des maladies";

- la croyance que la mise en ouvre de certaines activités de prévention de la maladie chez les femmes âgées (c.-à-d. faire de l'exercice, cesser de fumer) réduirait grandement leur qualité de vie, au point que le coût de la réduction du risque en dépasserait grandement les avantages;

- le défaut de reconnaître que les femmes âgées peuvent faire appel à des stratégies différentes de prévention de la maladie.

La possibilité que l'attitude et le comportement des professionnels de la santé envers les femmes âgées (c.-à-d. leurs attributions sur les symptômes et les maladies et leurs pratiques de diagnostic, de thérapie et de référence) puisse être influencée négativement par le statut socio-économique, l'âge et l'origine ethnique de leurs patientes âgées, augmente encore les préoccupations concernant les possibles inégalités dans les soins de santé dispensés aux femmes âgées. Une question particulièrement intéressantes pour les études futures consisterait à se demander si les femmes âgées en général, et plus particulièrement les femmes âgées provenant des groupes ethniques, sont plus susceptibles que d'autre groupes de bénéficier d'examens moins poussés, et d'un nombre moins grand d'options de traitement ou d'initiatives de prévention de la maladie, incluant les conseils sur les pratiques de dépistage et les changements aux habitudes de vie.

En résumé, le sommaire ci-dessus montre bien que, comme cela a souvent été souligné pour les personnes âgées en général, nos connaissances et notre compréhension des déterminants de la santé et du bien-être chez les femmes âgées ont été limitées par les résultats d'études qui n'ont pas pris en compte le caractère unique de cette population. Plus important encore, il est de plus en plus évident que l'absence relative d'études incorporant des analyses déterminées selon l'âge, le sexe et l'origine ethnique a contribué à l'adoption d'hypothèses fautives sur la pertinence de certaines questions de santé pour les femmes âgées et le potentiel d'intervention en promotion de la santé dans des sous-groupes particuliers 
au sein de cette population. Un objectif important des recherches futures dans ce domaine sera de déterminer et de mettre en ouvre des stratégies pour faciliter des travaux cliniques, de recherche et de politique visant la promotion d'une meilleure santé et de meilleurs soins pour l'ensemble des femmes âgées.

\section{Note}

* Cet éditorial se fonde sur le Livre blanc sur les questions de santé des femmes âgées, présenté à l'Association canadienne de gérontologie et comprenant des articles signés en collaboration par Huguette Léger, John P. Hirdes, Kimberly Ellis-Hale, Erin Y. Tjam, Iris Chi, Tina Wu et Paula C. Fletcher.

\section{Références}

Beery, T.A. (1995). Diagnosis and treatment of cardiac disease: Gender bias in the diagnosis and treatment of coronary artery disease. Heart and Lung, 24(6), $427-435$.

Bravo, G., Gauthier, P., Roy, P.M., Payette, H., Gaulin, P., Harvey, M., Péloquin, L., \& Dubois, M.F. (1996). Impact of a 12-month exercise program on the physical and psychological health of osteopenic women. Journal of the American Geriatrics Society, 44, 756-762.

Campbell, A.J., Spears, G.F., \& Borrie, M.J. (1990). Examination by logistic regression modelling of the variables which increase the relative risk of elderly women falling compared to elderly men. Journal of Clinical Epidemiology, 43(12), 1415-1420.

Chrischilles, E.A., Foley, D.J., Wallace, R.B., Lemke, J.H., Semla, T.P., Hanlon, J.T., Glynn, R.J., Ostfeld, A.M., \& Guralnik, J.M. (1992). Use of medications by persons 65 and over: Data from the established populations for epidemiologic studies of the elderly. Journal of Gerontology, 47(5), M137-M144.

Costanza, M.E. (1994). Issues in breast cancer screening in older women. Cancer, $74,2009-2015$.

Gee, E.M., \& Kimball, M.M. (1987). Women and aging. Toronto: Butterworths.

Graham, K., Carver., \& Brett, P.J. (1995). Alcohol and drug use by older women: Results of a national survey. La Revue canadienne du vieillissement, 14(4), $769-791$.

Gurwitz, J.H., Col, N.F., \& Avorn, J. (1992). The exclusion of the elderly and women from clinical trials in acute myocardial infarction. Journal of the American Medical Association, 268, 1417-1422.

Healy, B. (1995). A new prescription for women's health. New York: Viking Penguin.

Kiel, D.P. (1993). The evaluation of falls in the emergency department. Clinics in Geriatric Medicine, 9, 591-599.

Koenig, H.C., \& Blazer, D.G. (1992). Epidemiology of geriatric affective disorders. Clinics in Geriatric Medicine, 8, 235-252.

Lyness, J.M., Bruce, M.L., Koenig, H.G., Parmelee, P.A., Schulz, R., Lawton, M.P., \& Reynolds, C.F. (1996). Depression and medical illness in late life: Report of a symposium. Journal of the American Geriatrics Society, 44, 198-203.

Martin Matthews, A. (1991). Widowhood in later life. Toronto: Butterworths. 
Maxwell, C.J., \& Hirdes, J.P. (1993). The prevalence of smoking and implications for quality of life among the community based elderly. American Journal of Preventive Medicine, 9, 338-345.

Merkatz, R.B., Temple, R., Sobel, S., Feiden, K., Kessler, D.A., \& the Working Group on Women in Clinical Trials. (1993). Women in clinical trials of new drugs: A change in Food and Drug Administration policy. New England Journal of Medicine, 329(4), 292-296.

Nations Unies. (1995). Le Rapport mondial sur le développement humain. Cary, NY: Oxford University Press.

Nevitt, M.C., Cummings, S.R., \& Hudes, E.S. (1991). Risk factors for injurious falls: A prospective study. Journal of Gerontology, 46, M164-M170.

Riley, R. (Canadian Centre for Health Information, Statistics Canada) (1992). Accidental falls and injuries among seniors. Health Reports, 4(4), 341-344.

Rosenberg, L., Palmer, J.R., \& Shapiro, S. (1990). Decline in the risk of myocardial infarction among women who stop smoking. The New England Journal of Medicine, 322, 213-217.

Santé Canada. Le Groupe de travail fédéral-provincial-terretorial sur la santé des femmes. (1993). Un effort conjoint pour la santé mentale des femmes: Plan d'ensemble pour l'élaboration de politiques et de programmes. Ottawa: Santé Canada.

Sattin, R.W., Lambert-Huber, D.A., DeVito, C.A., Rodriguez, J.G., Ros, A., Bacchelli, S., Stevens, J.A., \& Waxweiler, R.J. (1990). The incidence of fall injury events among the elderly in a defined population. American Journal of Epidemiology, 131, 1028-1037.

Statistiques Canada. (1995). Women in Canada: A statistical report. Ottawa: Ministry of Industry Canada.

Young, J.E., Forbes, W.F., \& Hirdes, J.P. (1994). The association of disability with long-term care institutionalization of the elderly. La Revue canadienne $d u$ vieillissement, 13(1), 15-29. 